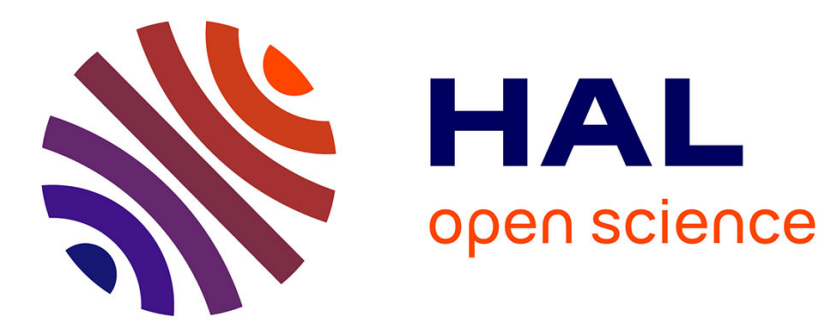

\title{
Investigations in the initial build-up stages of polyelectrolyte multilayers by laser reflectometry and atomic force microscopy
}

Séverine Diziain, J. Dejeu, L. Buisson, D. Charraut, F. Membrey, A. Foissy

\section{To cite this version:}

Séverine Diziain, J. Dejeu, L. Buisson, D. Charraut, F. Membrey, et al.. Investigations in the initial build-up stages of polyelectrolyte multilayers by laser reflectometry and atomic force microscopy. Thin Solid Films, 2007, 516 (1), pp.1-7. 10.1016/j.tsf.2007.04.036 . hal-00218239

\section{HAL Id: hal-00218239 \\ https://hal.science/hal-00218239}

Submitted on 21 Apr 2021

HAL is a multi-disciplinary open access archive for the deposit and dissemination of scientific research documents, whether they are published or not. The documents may come from teaching and research institutions in France or abroad, or from public or private research centers.
L'archive ouverte pluridisciplinaire HAL, est destinée au dépôt et à la diffusion de documents scientifiques de niveau recherche, publiés ou non, émanant des établissements d'enseignement et de recherche français ou étrangers, des laboratoires publics ou privés. 


\title{
Investigations in the initial build-up stages of polyelectrolyte multilayers by laser reflectometry and atomic force microscopy
}

\author{
S. Diziain ${ }^{\mathrm{a}, *}$, J. Dejeu $^{\mathrm{b}}$, L. Buisson ${ }^{\mathrm{a}}$, D. Charraut ${ }^{\mathrm{a}}$, F. Membrey $^{\mathrm{b}}$, A. Foissy $^{\mathrm{b}}$ \\ a Institut FEMTO-ST, Département d'Optique P.M. Duffeux, CNRS-UMR 6174, Université de Franche-Comté, 16 route de Gray, 25030 Besançon Cedex, France \\ ${ }^{\mathrm{b}}$ Laboratoire de Chimie des Matériaux et Interfaces, Université de Franche-Comté, 16 route de Gray, 25030 Besançon Cedex, France
}

This work aims to investigate the initial build-up stages of polyelectrolyte multilayers formed by the successive deposition of the weakly charged Poly(Allyl amine, $\mathrm{HCl}$ ), PAH and the strong acid Poly(Styrene Sulfonate), PSS, on silica at $\mathrm{pH}=9$. Two complementary tools were used: laser reflectometry to determine the step-by-step deposited weight of each polymer and Atomic Force Microscopy (AFM) to characterize the topography and measure the thickness of the film. The experiments show that the deposited weight of PAH is the same at each step (around $0.5 \mathrm{mg} . \mathrm{m}^{-2}$ ), whereas that of PSS increases more or less linearly along with the step number. Starting at the second polymer deposit (PSS), the structure of the film is very heterogeneous, with a thin polymer layer and bumps that are attributed to the presence of PSS/PAH hydrophobic complexes. The amount of bumps and their height were determined at each of the ten first polymer deposits. The film thickness between the bumps was evaluated by scratching small areas with an AFM tip. Our results support a former description of film growth, based on the electrostatic interactions between the two polymers and the substrate. They also reveal different the roles of the two polymers in the growth of the films: PAH is responsible for the progressive bi-dimensional coverage of the surface area and PSS for the increase in the thickness of the film.

Keywords: Laser reflectometry; Atomic Force Microscopy (AFM); Polyelectrolyte multilayers; Growth mechanism; Poly(Styrene Sulfonate); Poly(Allyl amine, $\mathrm{HCl}$ )

\section{Introduction}

The build-up of polyelectrolyte multilayers (PEM) on plane or particle surfaces has been widely studied in the last two decades, due both to the fundamental interest of scientists and to the large potential of applications [1]. PEM consists simply in the successive and alternate deposition of two oppositely charged polyelectrolytes. However difficult this technique may be, due to its numerous variables, its outcome is, nevertheless, very promising. Recent reviews on the subject show that many theoretical and practical aspects of the interaction between the two polymers along with the growth of the film remain ambiguous and inconclusive $[2,3]$. In order to improve control in the assembly of macromolecules, the main experimental

\footnotetext{
* Corresponding author. Tel.: +33 381 666382; fax: +33 381666423 .

E-mail address: severine.diziain@univ-fcomte.fr (S. Diziain).
}

factors (polymer charge densities, $\mathrm{pH}$, ionic strength, type of electrolytes) in film construction need to be better understood and chemical data, linked with morphological and structural characteristics. In a previous study, we addressed the influence of $\mathrm{pH}$ and salt concentration on the progressive weight uptake of alternating layers of a strong cationic polyelectrolyte, poly (dimethylaminoethyl methacrylate chloride), MADQUAT, and a weak polyacid, Poly(Acrylic Acid), PAA [4]. It was seen that much information could be drawn from a simple charge balance and that this reflects the contribution of electrolyte (counter) ions in the neutralization of the layer.

Our aim in the present work was to investigate the deposition and growth mechanisms in the earlier steps of the film construction up to 5 bilayers. The study involves a reflectometric monitoring of the successive deposited weights of the two polymers and a parallel analysis of the film morphology using Atomic Force Microscopy (AFM) studies. In order to take advantage of the numerous studies available in the literature, we 
chose a pair of polyelectrolytes commonly used in the build-up of PEMs, Poly(Allyl amine, $\mathrm{HCl}$ ) (PAH) and Poly(Styrene Sulfonate) (PSS). Investigations into the growth of PSS-PAH layers have led to contradictory results, advocating either linear growth (constant weight deposit at increasing step number) $[5,6]$ or exponential growth (the weight uptake of one polymer increases constantly in successive steps) $[7,8]$. In some cases, films grow exponentially at first and then linearly [9]. In all experimental studies, the growth rate of PEM depends on $\mathrm{pH}$, salt type and concentration, temperature, intermediate rinsing etc. Fundamentally, the growth of the PEM resides in the interaction forces between the two polyelectrolytes, which are electrostatic in nature, i.e. the growth is related to the charge densities and the charge-charge stoichiometry in neighboring polycation and polyanion layers. In general, it is thought that high ionization of polyions results in a lower growth rate and lower ionization gives thicker layers. Therefore, for weak polyelectrolytes, the $\mathrm{pH}$ is a determining parameter of the buildup mechanism, as are the type and the concentration of the salt [10]. In the present study the $\mathrm{pH}$ was set at 9, the PAH was slightly charged (ionization ratio $40 \%$ ) and the PSS, fully charged.

\section{Chemicals and experiments}

\subsection{Chemicals}

The sodium salt of Poly(4-Styrene Sulfonate) (PSS) with an average molecular weight of $70 \mathrm{kDa}$ was purchased from Alfa Aesar. PSS is a strong polyacid that is totally ionized in the whole range of $\mathrm{pH}$ used in this study [11]. The Poly(Allyl amine Hydrochloride) (PAH), obtained from Sigma Aldrich, has a similar average molecular weight. It is a weak polyelectrolyte, the ionization of which was measured by Petrov et al. [12]. The median ionization $\mathrm{p} K$ is 8.5 . At $\mathrm{pH}=9$ the ionization ratio (fraction of protonated amine groups) is $40 \%$. The ionic strength was adjusted by a sodium chloride solution used as received. The deposits were made on silica/silicon $(1,0,0)$ wafers

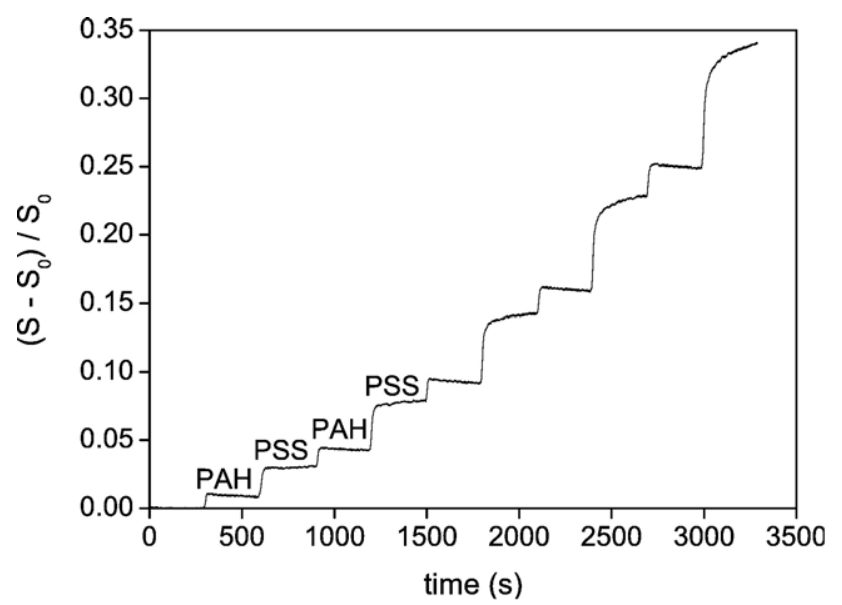

Fig. 1. Reflectometric signal for alternate deposition of PAH/PSS on a silica substrate at $\mathrm{pH}=9$ and with a ionic strength equal to $10^{-3} \mathrm{M}$.

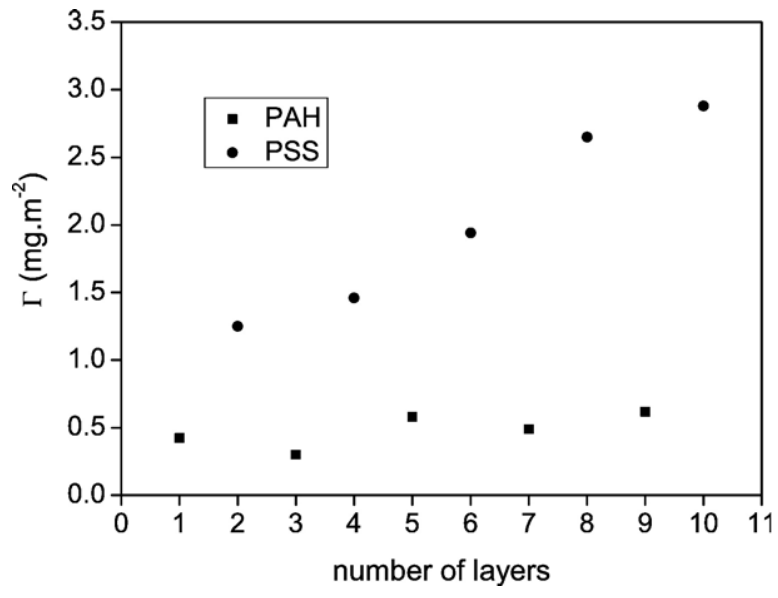

Fig. 2. Amount of deposited PAH and PSS in successive layers at $\mathrm{pH}=9$ and with a ionic strength equal to $10^{-3} \mathrm{M}$.

purchased from ACM (Villiers St. Frederic 78640, France). They were $150 \mathrm{~mm}$ in diameter and the silicon was $625 \pm 15 \mu \mathrm{m}$ in thickness. Substrates with two silica thicknesses (113 and $344 \mathrm{~nm}$ ) were used to calculate the deposited weight [13]. Silica thickness was measured with an ellipsometer (UVISEL-Jobin Yvon). Wafers were cleaned by immersion in a piranha solution ( 3 parts $\mathrm{H}_{2} \mathrm{SO}_{4}, 1$ part $\mathrm{H}_{2} \mathrm{O}_{2}$ ), rinsed and finally stored in Milli $\mathrm{Q}$ water. Each solution was prepared the day before and left in the reflectometer room over night for equilibration of temperature. The different concentrations were obtained by 10 successive dilutions. The $\mathrm{pH}$ of the solutions was controlled (and if necessary adjusted) just before the experiments.

\subsection{Deposition experiments and reflectometric analyses}

The polymer films were grown in the reflectometric cell described in detail previously [14]. Reflectometry delivers a signal that depends on the thickness and the refractive index of the adsorbed layer. In short, the relevant output of the reflectometer $S$, given by the equation $S=f \frac{R_{p}}{R_{s}}$, is an intensity ratio between the parallel $\left(R_{\mathrm{p}}\right)$ and the perpendicular $\left(R_{\mathrm{S}}\right)$ components of the polarized light beam that is reflected by the interface. The factor $f$ in the previous equation is an apparatus parameter. Practically, all experimental data are calculated as $\Delta S / S_{0}=\left(S-S_{0}\right) / S_{0}$ where $S$ and $S_{0}$ are the signal ratios with and without the adsorbed layer respectively ( $S_{0}$ is the reference signal). In the case of polyelectrolyte multilayers, the successive weight uptakes ( $\Gamma$ in $\mathrm{mg} \cdot \mathrm{m}^{-2}$ ) from the change of the reflectometric output were calculated with a previously described procedure [13]. Practically, the polymer solution $\left(100 \mathrm{mg} . \mathrm{L}^{-1}, 10^{-3} \mathrm{M} \mathrm{NaCl}\right)$ was introduced through a capillary set perpendicularly to the substrate (impinging jet cell). The reflectometric analyses were done at the stagnation point, at which the transport of molecules was purely diffusive. The liquid flow rate was $1.25 \mathrm{~cm}^{3} \mathrm{mn}^{-1}$. No water rinsing was done between polymer deposits. The deposition time was set at $5 \mathrm{mn}$, although the reflectometric output was constant after $2 \mathrm{mn}$. After each sample preparation, the wafer was dried under nitrogen and stored in a closed vessel. 


\subsection{AFM analyses}

AFM experiments were performed using a stand-alone SMENA scanning probe microscope (NT-MDT). AFM images were recorded on polymer films formed at the estimated stagnation point, in contact mode-more precisely in constant-force mode. During the scan, the deflection of the cantilever was maintained to a preset value by the feedback loop and the topographic map of the surface was obtained by the vertical displacement of the scanner. Conical silicon tips on the extremity of AFM cantilevers with a force constant from 0.1 to $0.3 \mathrm{~N} / \mathrm{m}$ were used to probe the samples (NT-MDT).
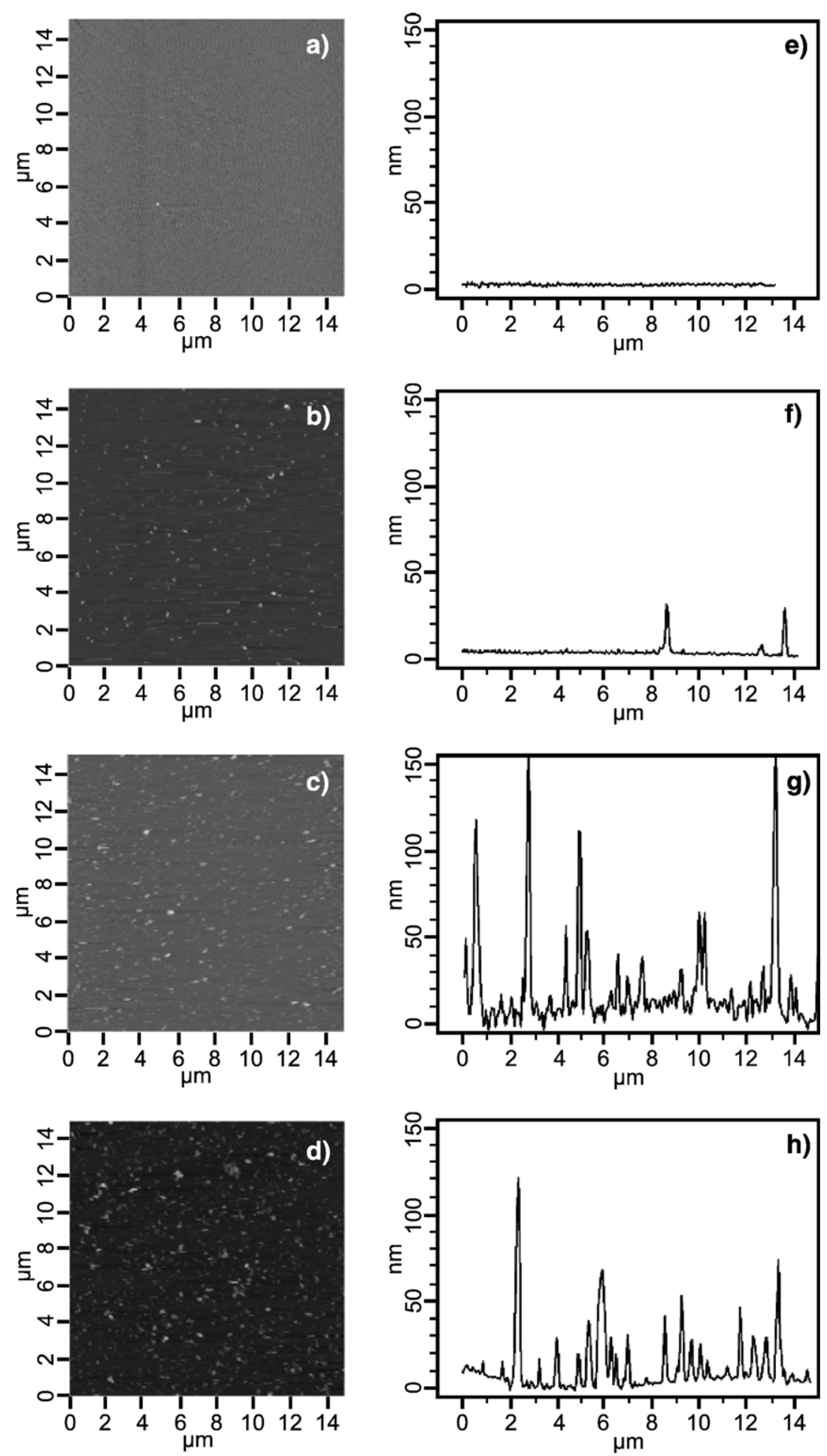

Fig. 3. AFM contact mode topographic images of PAH/PSS layers adsorbed on a silica substrate (dried samples) and the corresponding $z$-profiles along an arbitrary horizontal line. a), e): one layer; b), f): two layers; c), g): seven layers; d), h): eight layers. 


\section{Results}

\subsection{Depositions and reflectometric analyses}

The reflectometer output for the successive deposition strokes of polyelectrolytes is presented in Fig. 1. The first polymer layer was the cationic PAH, since the silica substrate was negatively charged. The steps in Fig. 1 show a steep increase upon polymer addition, followed by a plateau, which indicates that the adsorption reaction has been completed up to practical saturation. The graph also reveals different behaviour for PAH and PSS: PAH height remains roughly constant, whereas PSS height increases in successive steps.

The output was converted into deposited weight [13], which allowed the quantitative comparison presented in Fig. 2. The deposited weight of PAH was about $0.5 \mathrm{mg} \cdot \mathrm{m}^{-2}$ and that of PSS increased quite linearly between $1.25 \mathrm{mg} \cdot \mathrm{m}^{-2}$ and $2.58 \mathrm{mg} \cdot \mathrm{m}^{-2}$.

\subsection{AFM analyses}

Two series of samples, containing 1 to 10 successive polymer deposits (i.e. 5 bilayers), were submitted to AFM examination. One series was first dried (Nitrogen, room temperature) and the other one was kept wet for examination. The general morphology of several dried samples is presented in Fig. 3. It shows a rather regular distribution of islands, starting at the second layer (first PSS deposit), whereas the bare silica and the PAH covered silica substrate show no specific feature. Note the different scales in Fig. 3, between the $z$-axis of the profile and the horizontal line. Due to the $\simeq 60$ magnification of the $z$-axis the bump-like islands resemble peaks in the figure.

The average height of the bumps and their amount were determined as a function of the deposition number (Figs. 4 and 5) with a grain analysis software (Nova, NT-MDT). This analysis consisted in defining a threshold plane at a fixed distance $z=z_{0}$ to the base plane $(z=0)$. Each area above the threshold plane, corresponding to a closed domain, was interpreted as a separate bump. The height of the bumps was calculated from the $z_{0}$

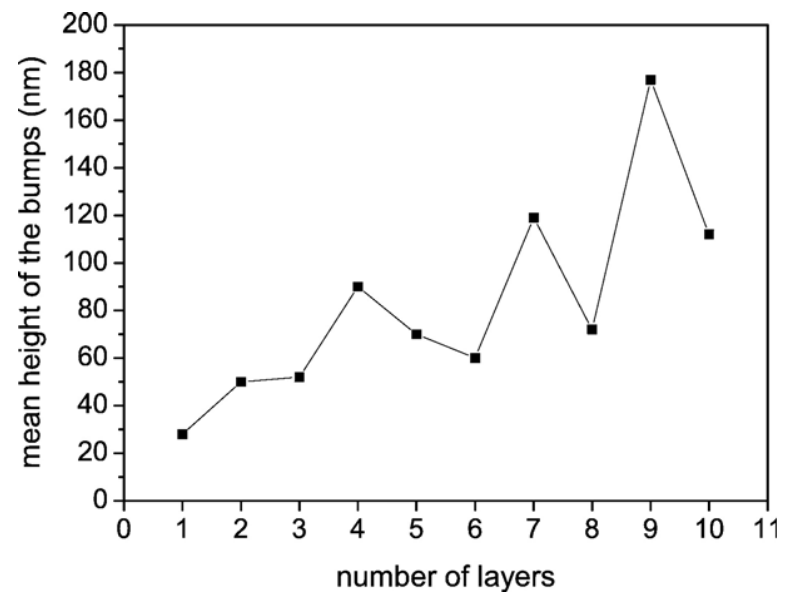

Fig. 4. Mean height of the bumps on the surface of PAH/PSS PEM as a function of the number of deposited layers.

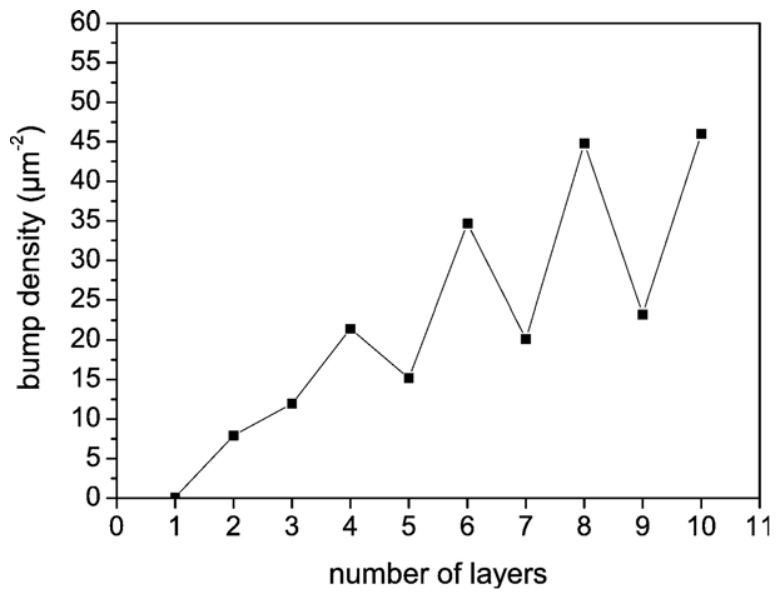

Fig. 5. Density of the bumps on the surface of PAH/PSS PEM as a function of the number of deposited layers.

position while the mean height of the bumps shown in Fig. 4 was the statistical average calculated from all bump heights on the sample. Bump number density (Fig. 5) was the grain number for which the height was above the threshold plane. Obviously, the measurements depend on the choice of the $z_{0}$ plane, which was
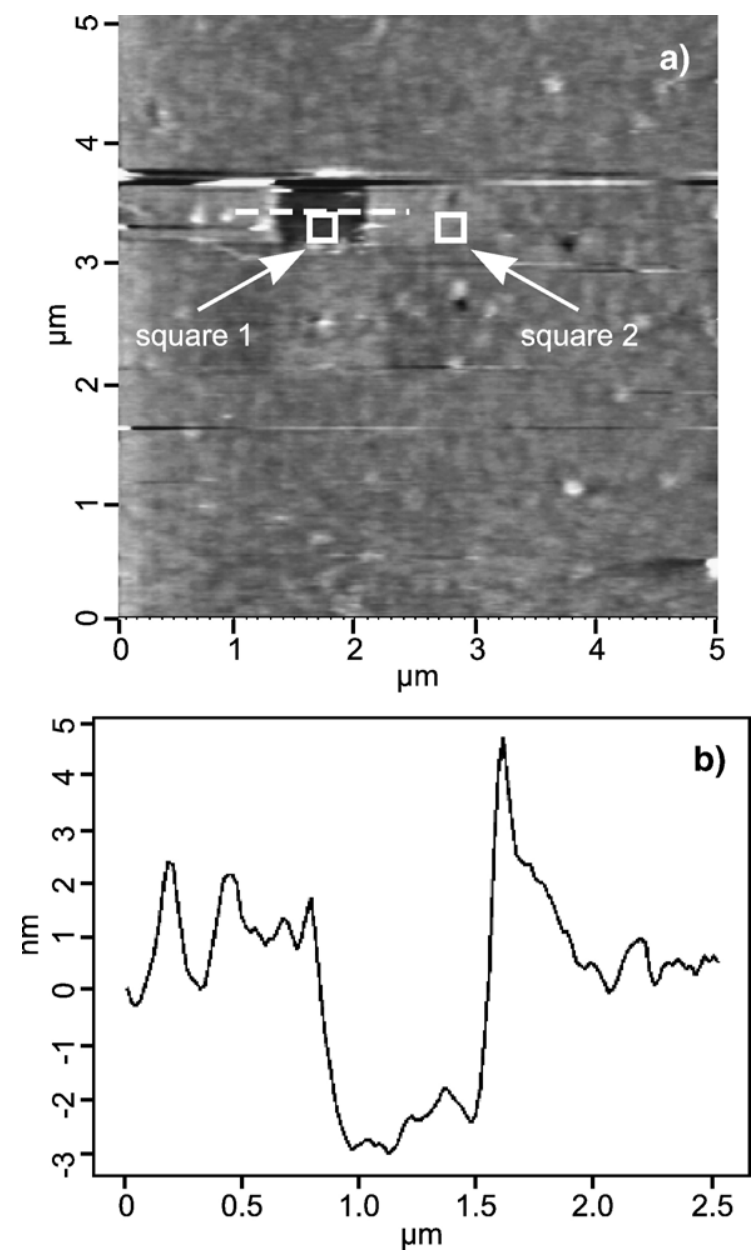

Fig. 6. a): AFM contact mode topographic images of five PAH/PSS layers after scratching a $(500 \times 500) \mu \mathrm{m}^{2}$ zone. b): $z$-profile above the scratched area (along the dashed line). 


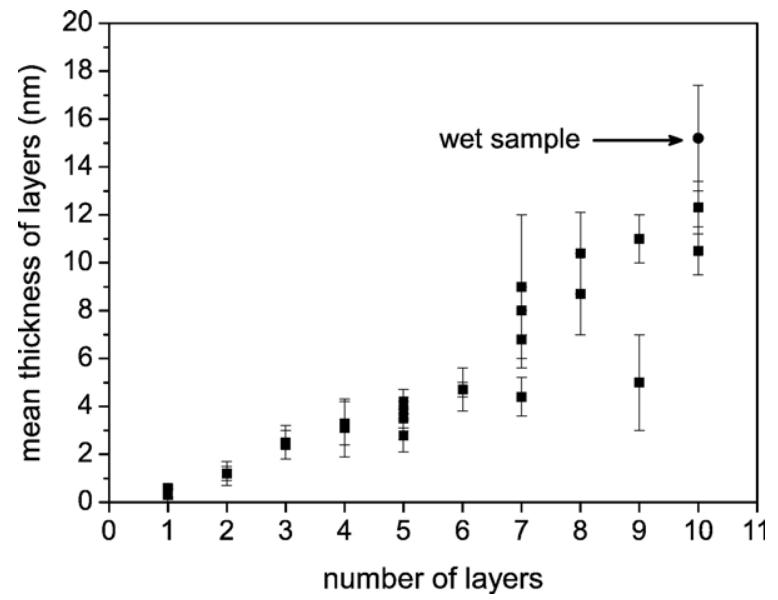

Fig. 7. Mean thickness of PAH/PSS PEM between bumps as a function of the number of deposited layers.

selected to avoid any artefact due to electronic noise from the AFM. Overall, the confidence interval of the measurements was estimated at about $15 \%$.

Figs. 4 and 5 show that both the average height and the bump density increase, but not regularly, with the number of polymer deposits. Minima in height at numbers 6,8 and 10 correspond to PSS as the last deposited polymer and maxima at numbers 7 and 9, to PAH. Height maxima correspond to minima for the bumps number density.

The scratching procedure described by Lobo et al. [15] was carried out to take off the polymer and measure the film thickness between the bumps. The method consists in "digging" an area of the film with the AFM tip until the substrate is reached. Scratching experiments were done on bare silica substrates, proving that the substrate itself does not suffer any abrasion by the tip. Practically, the chosen area (about $500 \times 500 \mathrm{~nm}^{2}$ ) was scanned repeatedly while increasing both the set point and the scan rate to their maximum value. After removing the polymer film, a larger zone containing the scratching area was scanned in contact mode, which allowed to measure the thickness of the film. Fig. 6 shows an example of a scratched area on a sample covered with 5 polyelectrolyte layers, and the topographical cross-section plotted along the dashed line. Quantitatively, the film thickness was calculated by a statistical procedure taking into account two areas for each sample; the relative mean heights were evaluated in the scratched area on the substrate (square 1 in Fig. 6) and on the polymer film (in square 2). The thickness of the layers results from the subtraction of these two values. The accuracy of this measurement is the sum of the root mean square values in both squares. Fig. 7 displays all measurements of the film thickness between the bumps, both for the wet and the dry samples. We see that a low amount of macromolecules adsorbed between the bumps and that the polymer film thickness increased more or less linearly at each successive deposit.

\section{Discussion}

The difference in the successive depositions of PAH and PSS shown in Figs. 1 and 2 resembles that for MADQUAT and PAA at $\mathrm{pH}=5.5$ [4], although in the latter case the progressive increase was observed for the polycation (MADQUAT), whereas the deposited weight for PAA was constant. An explanation for the inversion in the role of polymers in the growth phenomenon might be that the MADQUAT was fully charged, as was the PSS in the present study, and PAA was only charged at the ratio 0.25 , while the ionization ratio of $\mathrm{PAH}$ was 0.4 in the present experiment. In other words, the successive weights of deposited polymers might be more a question of charge density than of charge sign.

In a study addressing the early steps of build-up of PAH-PSS films on colloidal silica [10], we also found PSS to adsorb increasingly in successive steps at $\mathrm{pH}=9$, whereas $\mathrm{PAH}$ adsorbed at a roughly constant value $\left(0.8 \mathrm{mg}^{-\mathrm{m}^{-2}}\right)$. In this study, we suggested that PSS could penetrate the polycation-polyanion aggregates formed in former deposition layers; the driving force for this phenomenon was that the high charge density of the polymer enabled it capable to increase the ionization of PAH and therefore diffuse within the aggregates to unionized amine groups. Calculation of the charge balance after the successive deposition steps showed that the polyelectrolytes did not mutually neutralize their own charge, but that most of the PSS charge density was neutralized by co-adsorbed $\mathrm{Na}^{+}$counterions. The system certainly prefers charge-charge compensation between the polymers rather than with small ions, because it is much more favourable in terms of entropy increase. We also found that the $\mathrm{pH}$ increased significantly upon PSS adsorption on a former PAH layer [10], which was due to the increase in PAH ionization. Actually, the consumption of protons far exceeded the ionization capacity of the last deposited PAH layer, which proved that PSS reached earlier deposited PAH molecules. Several other works in the literature report that a so-called exponential film growth, with respect to the number of deposition steps, requires the diffusion of at least one of the two polymers within the layer [16]. Although this study was limited to the first deposited layers without reaching the whole covering of the substrate, the further build-up of the film could show up an exponential growth.

The present investigation delivers data that are a coherent complement to earlier studies. The general deposition scheme can be described as follows [10,16]. First the cationic PAH adsorbs on the silica substrate, up to the level where the positive charge excess prevents more macromolecules from reaching the surface (the adsorption kinetic limit of other polyelectrolytes is well documented; see Ref. [17] for example). At this adsorption stage most of the silica surface remains free of polymer. When PSS is circulated in the system, adsorption takes place specifically on the formerly deposited positive PAH molecules. It has been proven in separate experiments that PSS does not adsorb on bare silica [10]. Again, the PSS adsorption is limited by the overall charge excess of the film, which is negative at this point. Upon adsorption of PSS, PAH-PSS complexes form and aggregate on the surface, producing the bumps shown in AFM pictures. Further, when the sample is brought in contact with a new PAH solution, the PAH molecules may adsorb both on the aggregates and on the bare areas of the silica surface. At the stationary level, the overall charge of the film is positive, but the film contains two kinds of PAH molecules: some have adsorbed 
at the top of the aggregates and others have directly bound with the silica surface. When another new PSS solution is introduced, the molecules adsorb on the aggregates that have already formed, they also form new aggregates with isolated PAH molecules. They may also bridge several aggregates and molecules, and produce a coalescence phenomenon. Note that a very similar growing scenario was described by Picart et al. for a film composed of Poly(L-Lysine)/Hyaluronic Acid (PLL+/ $\mathrm{HA}^{-}$) on quartz [16]. Using optical waveguide lightmode spectroscopy and a quartz microbalance they found that positive PLL adsorbs over the entire surface of silica, whereas the next deposit of HA forms islands of PLL/HA complexes. They also observed the coalescence of islands and the increase in surface coverage during the successive and alternate deposits of the two polymers. In their case, the islands in the first bilayer were larger (a few micrometers width and height) than those we observe $(200 \mathrm{~nm})$. This difference may be due to the fact that they used high molecular weight polymers (about $200 \cdot 10^{5} \mathrm{Da}$ ) and that they made deposits in high ionic strength solutions.

The findings in the present study strengthen this description. In the course of the build-up we saw that the average bump height decreased significantly upon PSS adsorption and reversely with $\mathrm{PAH}$, which we attribute to the combination of two phenomena: the difference between PSS and PAH in the conformation of the outer polymer layer and the diffusion of PSS within the aggregates. Since PAH was only charged at $40 \%$ of full ionization, some segments of the macromolecules bound with the outermost sulfonate groups of PSS, and the remaining ones formed loops and tails that protruded into the solution, which caused the significant increase in film thickness. Conversely, the fully ionized PSS molecules laid flat on the surface with a stretched rod-like conformation. Upon contact with PSS, the charge density of PAH increased, which reduced the size of the loops and therefore the thickness of the outer layer. In addition, as stated earlier, PSS molecules diffused into the aggregates, which caused the bumps to shrink upon release of water and counterions. Similar shrinking or swelling phenomena, depending on the outermost polymer, were observed upon heating PSS-PAH films [18]. Kolasinska et al. also showed an alternating change in conformation and hydrophobic-hydrophilic properties of the external film layer for PAHPSS with water contact angle measurement [6].

Although the number of aggregates increased, on average, with the step number, Fig. 5 shows a slight decrease between the deposition of PSS and PAH. With PAH, all of the substrate was available for adsorption and the adsorption area remained roughly the same during the whole build-up process. The deposited weight was limited only by the electrostatic barrier raised by the excess in charge, which prevented molecules of the same charge from reaching the surface. This explains why the deposited weight of PAH was roughly constant $\left(0.5 \mathrm{mg} . \mathrm{m}^{-2}\right)$. In the case of PSS, adsorption took place only on the formerly deposited PAH molecules, some of which were at the top of the bumps and the others directly bound with the surface. Since we know that isolated PAH molecules were not detected by the AFM tip (Fig. 3a)), it is understandable that new bumps appeared only for each alternate deposition of PSS, since they formed new polymer complexes with the last deposited and isolated PAH molecules. Presumably, at the following PAH adsorption, the small decrease in the number of bumps was the result of a coalescence phenomenon induced by the binding of $\mathrm{PAH}$ with neighbouring polymer complexes.

We conclude that the growth of the polyelectrolyte multilayers proceeds via a complex mechanism in which the two macromolecules have a different role, depending essentially on their charge density and that of the substrate. Schematically, in the present case, $\mathrm{PAH}$, with a low charge density opposite that of the substrate, was responsible for the bi-dimensional increase in surface coverage, due to its adsorption both on the preformed polymer complexes and on the bare surface. Conversely, the highly charged PSS caused thickening and densification of the layer, thus increasing the number of bumps and the weight uptake, both of which are explained by the formation of hydrophobic complexes and the diffusion of PSS into the aggregates.

\section{Conclusion}

Our work addressed the initial build-up stages of polyelectrolyte multilayers made on silica with a strong (highly charged) and a weak (low charged) polyelectrolyte, PSS and PAH respectively. Two complementary analyses were done - a stepby-step measurement of the adsorbed polymer weight (by reflectometry) and the evolution of the film morphology (by AFM). Together, these analyses demonstrated a comprehensive mechanism of the early growth, with each polymer behaving differently. The deposited weight of PSS increased constantly from step to step, whereas that of PAH remained roughly constant. While PAH could adsorb on the entire surface of silica, PSS could only form hydrophobic aggregates with the pre-adsorbed $\mathrm{PAH}$ molecules. PAH formed an outermost polymer layer with loops and tails protruding in the solution, but the rod-like PSS laid flat on the surface and diffused into the preformed aggregates. These differences complement earlier investigations in which a $\mathrm{pH}$ drift was observed towards the acidic or the basic direction respectively, depending on which of the two polymers was adsorbed last [10], small counterions were co-adsorbed (with PSS) or not (with PAH) [10], the film was hydrophilic (PSS) or hydrophobic (PAH) [6] and the film shrank more (PAH) or less (PSS) upon heating [18]. With regards to film growth, each polymer had a specific role: $\mathrm{PAH}$ was responsible for the increase in the surface coverage by adsorbing on the remaining bare silica areas whereas PSS determined the thickening and densification of the film by forming polymer complexes with $\mathrm{PAH}$ and by diffusing into the polymer aggregates. These studies provide tools to rationalize the choice of the physico-chemical parameters in order to control the growth and the properties of PEM.

\section{Acknowledgments}

This work was supported by the EU under contract NMP4CT-2003-001428: Nano-capsules for Targeted Controlled Delivery of Chemicals and by a CNRS grant. 


\section{References}

[1] G. Decher, Science 277 (1997) 1232.

[2] B. Bertrand, A. Jonas, R. Legras, Macromol. Rapid Commun. 21 (2000) 319

[3] M. Schönhoff, Curr. Opin. Colloid Interface Sci. 8 (2003) 86.

[4] C. Buron, C. Filiâtre, M. Membrey, H. Perrot, A. Foissy, J. Colloid Interface Sci. 296 (2006) 409.

[5] V. Bosio, F. Dubreuil, G. Bogdanovic, A. Fery, Colloids Surf., A Physicochem. Eng. Asp. 243 (2004) 147.

[6] M. Kolasinska, P. Warszynski, Appl. Surf. Sci. 252 (2005) 759.

[7] P. Lavalle, C. Gergely, F. Cuisinier, G. Decher, P. Schaaf, J. Voegel, C. Picart, Macromolecules 35 (2002) 4458.

[8] J. Ruths, F. Essler, G. Decher, Riegler, Langmuir 16 (2000) 8871.

[9] M. Michel, A. Izquierdo, G. Decher, J.-C. Voegel, P. Schaaf, V. Ball, Langmuir 21 (2005) 7854.

[10] J. Dejeu, L. Buisson, M.C. Guth, C. Roidor, F. Membrey, D. Charraut, A. Foissy, Colloids Surf., A Physicochem. Eng. Asp. 288 (2006) 26.
[11] M. Balastre, J. Persello, A. Foissy, J. Argillier, J. Colloid Interface Sci. 219 (1999) 155.

[12] A. Petrov, A. Antipov, G. Sukhorukov, Macromolecules 36 (2003) 10079.

[13] C.C. Buron, F. Membrey, C. Filiâtre, A. Foissy, Colloids Surf., A Physicochem. Eng. Asp. 289 (2006) 163.

[14] T. Roques-Carmes, F. Membrey, C. Filiâtre, A. Foissy, J. Colloid Interface Sci. 245 (2002) 257.

[15] R.F.M. Lobo, M.A. Pereira-da Silva, M. Raposo, R.M. Faria, O.N.O. Jr., M.A. Pereira-da Silva, R.M. Faria, Nanotechnology 10 (1999) 389.

[16] C. Picart, P. Lavalle, P. Hubert, F. Cuisinier, G. Decher, P. Schaaf, J.-C. Voegel, Langmuir 17 (2001) 7414.

[17] M. Cohen Stuart, C. Hoogendam, A. de Keizer, J. Phys.: Condens. Matter 9 (1997) 7767.

[18] S. Leporatti, C. Gao, A. Voigt, E. Donath, H. Möhwald, Eur. Phys. J., E Soft Matter 5 (2001) 13. 